\title{
Review: inhaled corticosteroids alone appear to be as effective as oral corticosteroids after emergency department discharge for acute asthma
}

\author{
Edmonds ML, Camargo CA Jr, Saunders LD, et al. Inhaled steroids in acute asthma following emergency department \\ discharge. Cochrane Database Syst Rev 2000;(3):CD002316 (latest version 21 March 2000).

\section{QUESTION: Are inhaled corticosteroids (ICSs) alone or combined with oral corticosteroids (OCSs) as effective as OCSs alone after discharge from the emergency department (ED) for acute asthma?}

\section{Data sources}

Published and unpublished trials were identified by searching the Cochrane Airways Review Group "Asthma and wheeze" randomised controlled trial register (to April 1999), which is based on searches of EMBASE/Excerpta Medica, Medline, CINAHL, and the Cochrane Clinical Trials Register; handsearching 20 respiratory care journals, abstracts from 3 respiratory societies, and meeting abstracts from the American Thoracic Society; reviewing bibliographies of retrieved studies; and contacting experts and pharmaceutical companies.

\section{Study selection}

Randomised or quasirandomised controlled trials published in any language were selected if they included patients who were discharged from an ED after treatment for acute asthma and if patients were allocated to ICS treatment (ie, any corticosteroid agent administered by metered-dose inhaler, other inhaler, or nebuliser) in addition to or as a substitute for OCS treatment.

\section{Data extraction}

Data were extracted on study methods, patient characteristics, interventions, and outcomes. Main outcomes were acute asthma relapse and asthma specific quality of life. Methodological quality of studies was assessed using the Cochrane approach and the Jadad criteria.

\section{Main results}

10 studies (6 unpublished, 6 involved adults) met the inclusion criteria: 3 compared ICSs plus OCSs with OCSs alone (909 patients), and 7 compared ICSs alone with OCSs alone (1204 patients). All studies had quality scores $\geqslant 4$ out of 5 . Follow up for individual trials ranged from $70 \%$ to $100 \%$. Meta-analyses of studies that compared ICSs plus OCSs with OCSs alone found no differences between groups at either 7-10 days or 20-24 days for asthma relapse (3 studies), quality of life (2 studies), asthma symptoms ( 2 studies), $\beta$ agonist use ( 3 studies), side effects (2 studies), hospital admission (2 studies), or pulmonary function tests (2 studies). Meta-analyses of studies that compared ICSs alone with OCSs alone found no differences between groups for asthma relapse at $7-10$ days (4 studies) or 16-21 days (2 studies), quality of life (2 studies), $\beta$ agonist use at 7-10 days (2 studies), peak expiratory flow rates (PEFRs) at 7-10 days (6 studies), or percentage of predicted PEFR at either time interval. At 20-24 days, the ICS group had a higher PEFR than did the control group (weighted mean difference $15.2 \mathrm{l} / \mathrm{min}, 95 \%$ CI 2 to 29).

\section{Conclusions}

Inhaled corticosteroids alone appear to be as effective as oral corticosteroids after discharge from the emergency department in patients with mild asthma exacerbations. Evidence is insufficient on the benefit of the addition of inhaled corticosteroids to oral corticosteroids in this setting.

\section{COMMENTARY}

Many controlled trials have established the efficacy of ICSs in chronic asthma. The role of ICSs in acute asthma remains controversial. The well done meta-analysis by Edmonds et al reviews the use of ICSs alone or in combination with OCSs in the setting of acute exacerbations of asthma. The study set out to answer 2 questions: are ICSs beneficial when added to the standard short course treatment with OCSs, and can ICS treatment be substituted for OCS treatment after an acute asthma attack?

Three trials involving almost 1000 patients addressed the first question. Both groups received a fixed dose of oral prednisone for 5-7 days. The addition of ICSs did not reduce asthma relapse, despite a trend in favour of ICSs. However, the length of follow up in these 3 studies was 20-24 days, and several studies have shown that reduction in airway hyperresponsiveness occurs over several weeks with ICSs and may not be maximal for $\geqslant 3$ months in some patients..$^{1-3}$

The 7 studies that addressed the second question involved $>1200$ patients but included only patients with relatively mild asthma and varied markedly in their reported outcomes. No differences were found between treatments for asthma relapse at either 7-10 days or 16-21 days. Although 6 of the 7 trials concluded that ICSs could be substituted for OCSs, the authors' power calculations suggest that these data are not sufficient to conclude that the 2 treatments are equivalent.

Until further research results are available, the mainstay of treatment for acute asthma exacerbations is still 5-10 days of OCSs. ICSs are a reasonable alternative in patients with mild asthma who are at high risk for complication from OCSs. The addition of ICSs to OCS treatment is reasonable, yet at present the benefits are unproved and must be individualised for each patient.

Jay Peters, MD

University of Texas, San Antonio, Texas, USA

1 Juniper EF, Kline PA, Vanzieleghem MA, et al. Effect of long-term treatment with an inhaled corticosteroid (budesonide) on airway hyperresponsiveness and clinical asthma in nonsteroid-dependent asthmatics. Am Rev Respir Dis 1990;142:832-6.

2 van Essen-Zandvliet EE, Hughes MD, Waalkens HJ, et al. Effects of 22 months of treatment with inhaled corticosteroids and/or beta-2-agonists on lung function, airway responsiveness, and symptoms in children with asthma. The Dutch chronic non-specific lung disease study group. Am Rev Respir Dis 1992;146:547-54.

3 Kerstjens HA, Brand PL, Hughes MD, et al. A comparison of bronchodilator therapy with or without inhaled corticosteroid therapy for obstructive airways disease. Dutch chronic non-specific lung disease study group. $N$ Engl $J$ Med 1992;327:1413-9 